\title{
The Analysis of a Model of Water Temperature in Space and Time
}

\author{
Wenyan Dong \\ School of Electrical \& Electronic Engineering, North China Electric Power University, Baoding \\ 071003, China. \\ dongwenyan666@163.com
}

Keywords: Heat conduction differential; Fourier's law; newton cooling law.

\begin{abstract}
In this paper, the change of water temperature in the bathtub is mainly studied. Through the simulation of the water temperature distribution in time and space and the construction of the thermal conduction model, we discuss the water injection scheme. According to the Fourier's law and Newton cooling law, we establish a one-dimensional heat conduction differential model with water temperature, bath depth and time as variables. Then the model is classified into two parts because of the heat source's existence. By the evaporation model we can conclude the relationship between the water temperature and time, then solve the partial differential equation in the usage of implicit format of finite difference method. Equation of the water speed and temperature and the optimal scheme can be determined through the Improved Genetic Algorithm on the base of law of conservation of energy. The judging equation can be obtained after the analysis of the various influence factors of the strategy plan. Finally, we draw a conclusion that: the shape and volume of bathtub infect the strategy most.
\end{abstract}

\section{Introduction}

In today's world, a lot of people like taking a bath after work. But there is a disturbing situation:

A person fills a bathtub with hot water from a single faucet and settles into the bathtub to cleanse and relax. Unfortunately, the bathtub is not a spa-style tub with a secondary heating system and circulating jets, but rather a simple water containment vessel. After a while, the bath gets noticeably cooler, so the person adds a constant trickle of hot water from the faucet to reheat the bathing water. The bathtub is designed in such a way that when the tub reaches its capacity, excess water escapes through an overflow drain.

Develop a model of the temperature of the bathtub water in space and time to determine the best strategy the person in the bathtub can adopt to keep the temperature even throughout the bathtub and as close as possible to the initial temperature without wasting too much water.

\section{Analysis}

The problem asks us to develop a model of the temperature of the bathtub water in space and time, so we should build a heat conduction equation of the object in the general situation, and then utilize it in the bath water temperature model. In the process of building the model, we think we need to depart the whole bathing process into two parts: before adding hot water to reheat and after that. The reason is that there is no heat source in the first process, but there exists hot source in the latter process. Then we can solve the two models with different initial and boundary conditions to build the model of the temperature of the bathtub water in space and time.

In order to determine the best strategy the person in the bathtub can adopt to keep the temperature even throughout the bathtub and as close as possible to the initial temperature without wasting too much water, we must use the water temperature model. Because we have got the function which flow and time are for temperature, we can optimal scheme for the person to add the hot water.

\section{Assumptions}

1. The base of bathtub does not exchange heat with water. 
2. The room temperature is almost constant,the most comfortable temperature for a person is $25^{\circ} \mathrm{C}$.

3. Before the person settle into the bath, the temperature is almost fixed.

\section{Symbol Definitions}

\begin{tabular}{cl}
\hline Symbols & Definitions \\
\hline$\Phi$ & Heat source \\
$y$ & Abscissa \\
$z$ & Ordinate \\
$c$ & Height \\
$\rho$ & Specific heat capacity \\
$\lambda$ & Density \\
$a$ & Heat conductivity \\
$T$ & Thermal diffusivity \\
$t$ & The temperature of the water \\
$f_{1}$ & The time after filling the bathtub \\
$f_{2}$ & The flow of hot water added into the bathtub \\
$m$ & the quality of the bath water at the initial time \\
$S_{1}$ & the area of bath crock walls surface \\
$S_{2}$ & the area of bathtub upper surface \\
$H$ & The height of bathtub \\
$\mu$ & The coefficient of the impact which the person in the bathtub has on the \\
$d$ & change of water temperature at unit time \\
$h$ & The thickness of the tub wall \\
\end{tabular}

\section{The Water Temperature Change Model}

In order to obtain the mathematic expression of the object temperature field of thermal conductivity, we can build the temperature change expressions which are called differential equation of heat conduction on the base of the law of conservation of energy and Fourier law.

First, we can take an infinitesimal parallelepiped arbitrarily from the object of thermal conductivity to analyze energy equilibrium of the infinitesimal. If there is a heat source in the object, which is seats $\Phi$, it stands for the heat energy to produce or consume at the unit time in the unit volume. If the energy is produced, $\Phi$ is positive, else it is minus. We assume that the physical property of an object is a function of temperature.

The heat flux at any direction in the space can break up into departed heat flux $\Phi_{x^{y}} \Phi_{y^{y}} \Phi_{z}$ at $x_{y}, y, z$ coordinate orientations. According to Fourier law,the heat fluxes which are guided into infinitesimals through the surfaces of $x=x, y=y, z=z$ infinitesimals can be written as follows:

$$
\left\{\begin{array}{l}
\left(\Phi_{x}\right)_{x}=-\lambda\left(\frac{\partial T}{\partial x}\right)_{x} d y d z \\
\left(\Phi_{y}\right)_{y}=-\lambda\left(\frac{\partial T}{\partial y}\right)_{y} d x d z \\
\left(\Phi_{z}\right)_{z}=-\lambda\left(\frac{\partial T}{\partial z}\right)_{z} d x d y
\end{array}\right.
$$

Among those formula, $\left(\Phi_{x}\right)_{x}$ stands for the value of $\Phi_{x}$ at the point $\mathrm{x}$. The rest can be analogized according to the first one.

According to the law of conservation of energy, there exists a thermal equilibrium relationship at any time interval for the infinitesimal:

$$
\Delta Q_{1}+E_{1}=\Delta Q_{2}+\Delta E
$$


In the formula, $\Delta Q_{1}$ stands for the total flux guided into the infinitesimal, $E_{1}$ stands for the formation heat of the heat source in the infinitesimal, $\Delta Q_{2}$ stands for the total flux exported out of the infinitesimal, $\Delta E$ stands for the incremental of the infinitesimal'sinternal energy.

In the formula (2),

$$
\begin{aligned}
& \Delta Q_{2}=\rho c \frac{\partial T}{\partial t} d x d y d z \\
& E_{2}=\Phi d x d y d z
\end{aligned}
$$

Substitute the formula (1)(3)(4) into the formula (3), we can get the general form of the three-dimensional differential equation of heat conduction:

$$
\rho c \frac{\partial T}{\partial t}=\frac{\partial}{\partial x}\left(\lambda \frac{\partial T}{\partial x}\right)+\frac{\partial}{\partial y}\left(\lambda \frac{\partial T}{\partial y}\right)+\frac{\partial}{\partial z}\left(\lambda \frac{\partial T}{\partial z}\right)+\Phi
$$

In that formula, $\rho, c, \lambda, \Phi$ can be variable.

In order to study the situation that the water temperature changes along with the change of time and space, we divide the whole process into two stages:

Firstly, we consider that before the person adds hot water to reheat the bath water, the fringe effect can be ignored on the base of the searching information. At this condition, the temperature of water is just a function of time and depth of the water, and then we can obtain a differential equation as follows:

$$
\rho c \frac{\partial T}{\partial t}(z, t)=\lambda \frac{\partial^{2} T}{\partial z^{2}}(z, t)
$$

In the condition of natural heat dissipation, we can the initial condition and boundary conditioncorresponding to the equation:

$$
\left\{\begin{array}{c}
T(z, 0)=T_{0} \\
\frac{\partial T}{\partial n}=-\frac{h}{\lambda}\left(T_{1}-T_{2}\right)
\end{array}\right.
$$

In the above formulas, $T_{1}$ stands forthe temperature of water in contact with the air, $T_{2}$ stands for the temperature of external environment.

Considering theone-dimensional boundary value problem of heat conduction equation, we adopt high precision implicit difference schemeto solve it:

Take the space steps $\Delta z=z_{z} \Delta t=\tau$, subdivision regions $G$, nodes $\left(z_{j}, t_{k}\right)$,shorthand as $(j, k)$.

Considering the discrete definite solution problem at the node $(j, k)$, westructure a difference operator as follows:

$$
\begin{gathered}
L_{k}[T]_{j}^{k} \equiv \varepsilon_{1} \frac{T_{j-1}^{k}-T_{j-1}^{k-1}}{\tau}+\varepsilon_{2} \frac{T_{j}^{k}-T_{j}^{k-1}}{\tau}+\varepsilon_{3} \frac{T_{j+1}^{k}-T_{j+1}^{k-1}}{\tau}-\varepsilon_{4} a \frac{T_{j+1}^{k}-2 T_{j}^{k}+T_{j-1}^{k}}{h^{2}} \\
-\varepsilon_{5} a \frac{T_{j+1}^{k-1}-2 T_{j}^{k-1}+T_{j-1}^{k-1}}{h^{2}}
\end{gathered}
$$

In the formula, $\varepsilon_{k}(k=1,2, \cdots 5)$ are undetermined parameters. When we select these parameters properly, the difference equation(8) can approach the differential equation(6) closely. Then it will have truncation error of higher order as soon as possible, and have better stability.

When the solution of the differential equation (6) is sufficiently smoothly, the following formula is established:

$$
\frac{\partial^{m+n} T}{\partial z^{m} \partial t^{n}}=a^{n} \frac{\partial^{m+2 n} T}{\partial z^{m+2 n}} \quad m, n \text { are nonnegative integers }
$$

After our calculation, we get the following difference scheme:

$$
\begin{aligned}
(1-6 \tau) T_{j+1}^{k} & +(10+12) T_{j}^{k}+(1-6 \tau) T_{j-1}^{k} \\
& =(1+6 \tau) T_{j+1}^{k-1}+(10-12) T_{j}^{k-1}+(1+6 \tau) T_{j-1}^{k-1}
\end{aligned}
$$

That is implicit scheme on the second floor, its truncation error is $\mathrm{O}\left(\tau^{3}+h^{4}\right)$.

At last, we get the analytical solution of the model by using the method of separation of variables:

$$
\begin{aligned}
& \mathrm{T}=T_{0}+\left(T_{0}-T_{\infty}\right) \sum_{n=1}^{\infty} \frac{2 \sin \mu}{\mu+\sin \mu \cos \mu} \cos \left(\mu \frac{z}{L}\right) e^{-\mu^{2} F_{0}} \\
& F_{0}=\frac{a \pi}{L^{2}}, a=\frac{h}{c \rho} \mu
\end{aligned}
$$


After analyzing the boundary conditions, we can get change of the water surface temperature as time goes by.

When the bath gets noticeably cooler, the personadds a constant trickle of hot water from the faucet to reheat the bathing water. According to the design principle of the bath that when the tub reaches its capacity, excess water escapes through an overflow drain, we can draw a conclusion that water volume added into the bathtub equals the water volume escaped from the tub in the bathing process.

There is an import tube and an outlet pipe. We can make the temperature of the water in the bathtub through the control of some factors, such as the flow of the import tube. Obviously, the heat of water in the bathtub and the outside world will exchange energy by heat conduction, convection and radiation. Therefore, we can draw a conclusion that the change of the bathtub is made up of the added water's release of heat energy and the heat loss from the bathtub. Among these, the added water's release of heat energy refers to the difference value between the heat that the added water brought into the tub at a certain time and the heat energy taken away by the discharged bath water. In this case, we can build the model II:

$$
\left\{\begin{array}{c}
c m \frac{d T}{d t}=f_{1} \rho c\left(T_{3}-T\right)-\mathrm{HS}_{2}\left(T-T_{2}\right)-\mu \\
T(t=0)=T_{0}{ }^{\prime}
\end{array}\right.
$$

In the above formulas, $\mathrm{T}_{1}$ stands for the temperature of the added water, $\mathrm{T}_{2}$ stands for the temperature of external environment.

Due to there is no such apparent difference between the Model I and Model II, we can take the similar method and steps to solve the second model. Finally, we get the solution of the model:

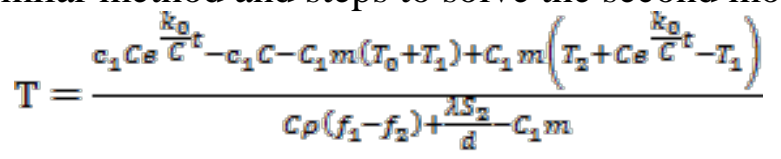

\section{Summary}

We draw the following conclusion that the main factors which can influence the optimal strategy are the volume and the upper surface of the bathtub, motion amplitude of the person in the water, and the contact area which the body is exposed to air.

\section{References}

[1]. Honglie Sun. The High Accuracy Implicit Difference scheme for solving Parabolic Equations of One-Dimension. MATHEMATICS IN PRACTICE AND THEORY. Vol.28 (1998) No. 3, p. 197-200.

[2]. Baosheng Cui, Xuean Xu. STATIC STATE WATER THERMAL MODEL STUDY. REMOTE SENSING FOR LAND \& RFSOURCFS. Vol.15 (1992) No. 12, p. 50-53.

[3]. Zhihui Chen. One-Dimensional Unsteady Heat Conduction Question of Variable Physical Properties With Finite Differential Method. JOURNAL OF SHENYANG INSTITUTE OF GOLD TECHNONGY. Vol.16 (1997) No. 2, p. 121-128. 